\title{
Evaluation of GeneXpert MTB/RIF assay in diagnosis of extra pulmonary tuberculosis and rifampicin resistance
}

\author{
Jagadevi $^{1}$, Sumanta A. ${ }^{2}$, Shubha D.S. ${ }^{3}$, Sudhindra K.S. ${ }^{4}$, Sangolli B. ${ }^{5}$ \\ ${ }^{1}$ Dr. Jagadevi, Assistant Professor, ${ }^{2}$ Dr. Sumanta A, Assistant Professor, ${ }^{3}$ Dr. Shubha D S., Professor and Head, \\ ${ }^{4}$ Dr. Sudhindra K.S., Professor, all authors are affiliated with Department of Microbiology, ${ }^{5}$ Dr. Basavaraj Sangolli, \\ Assistant Professor, Department of TB \& Chest, all authors are affiliated with Basaveshwara Medical College \& \\ Hospital, Chitradurga, India.
}

Corresponding Author: Dr. Sumanta A., Assistant Professor, Department of Microbiology, Basaveshwara Medical College \& Hospital. SJM Campus, NH-4 Bypass, Chitradurga, Karnataka. E-mail: drasumant@gmail.com

\begin{abstract}
Background: The prevalence of tuberculosis is still on the rise particularly extrapulmonary cases. There is an urgent need for rapid diagnosis of these cases for prompt initiation of treatment. Aim: To evaluate the role of GeneXpert MTB/RIF Assay in the diagnosis of clinically suspected extrapulmonary tuberculosis (EPTB) and to detect rifampicin resistance in these cases. Materials and methods: A total of 241 samples from April 2016 to July 2018, from all clinically suspected EPTB patients with support of either laboratory or radiological evidence were included in study. 132 pleural fluid, 59 lymph node aspirate, $21 \mathrm{CSF}, 17$ pus, 9 ascitic fluid and 3 synovial fluid samples were screened for presence of acid fast bacilli (AFB) by conventional Zeihl-Neelsen (ZN) technique. The same samples were also used for testing by GeneXpert MTB/RIF Assay. Results: Out of 241 EPTB samples tested, none were positive for AFB by ZN staining. Overall $9.54 \%$ (23 out of 241) samples were positive for MTB by GeneXpert with a positivity rate of $23.80 \%$, $13.55 \%, 11.76 \% \& 6.06 \%$ respectively for CSF, lymph node aspirate, pus and pleural fluid. MTB was not detected in any of ascitic fluid \& synovial fluid samples. Of the 23 MTB positive samples detected by GeneXpert, all were sensitive to rifampicin. Conclusions: GeneXpert was found to be simple, effective and useful diagnostic method for detection of EPTB due to its rapidity \& simultaneous detection of rifampicin resistance, thus reducing the time taken for initiation of treatment.
\end{abstract}

Key words: Extrapulmonary tuberculosis (EPTB), Mycobacterium tuberculosis (MTB), GeneXpert MTB/RIF (GeneXpert), Rifampicin (RIF) resistance,

\section{Introduction}

Approximately one third of the world's population has been infected with M.tuberculosis (MTB) and is at risk of developing the disease later in life. The killer disease thus claims more than five thousand deaths everyday i.e., one every fifteen seconds [1]. Despite many advances in its diagnosis and treatment, problem of tuberculosis is on the rise, both globally and in India.

Tuberculosis is usually a pulmonary disease; extrapulmonary tuberculosis (EPTB) is much less common, but infection may occur in any organ or tissue including lymph nodes, meninges, pleura, pericardium, kidneys, bones, joints, larynx, skin, peritoneum, intestine and eyes. EPTB constitutes $20 \%$ of burden of TB globally. EPTB is a paucibacillary disease as the number of

Manuscript received: $4^{\text {th }}$ October 2018

Reviewed: $14^{\text {th }}$ October 2018

Author Corrected: $20^{\text {th }}$ October 2018

Accepted for Publication: $25^{\text {th }}$ October 2018 bacteria is less to detect and are located deep seated in the organs [2]. The most effective control measure to check the spread of tuberculosis is to detect early to treat optimally at the earliest. Although Ziehl-Neelsen (ZN) stain - smear microscopy is most commonly employed for early detection, it is rather insensitive and fails to detect large number of cases [3].

Conventional methods like histology are never diagnostic and culture methods such as culture and drug susceptibility testing is complex and requires 6-8 weeks. Such a prolonged turnaround time is unacceptable for both medical and epidemiological purpose [4]. Though better methods of diagnosis of tuberculosis by molecular techniques are available; they are not being used in many cases owing to high cost per test or non-availability at majority of the centers. Hence there is a need for newer and faster diagnostic methods 


\section{Original Research Article}

like nucleic acid amplification techniques (NAAT). Cartridge based nucleic acid amplification techniques (CBNAAT) such as GeneXpert have been recently recommended by World Health Organization and introduced in the diagnostic algorithms of tuberculosis. This GeneXpert, also known as Xpert MTB/ RIF assay or Xpertassay makes use of five different molecular beacons and each one of them being labelled with a differentially coloured flourophore and responding to a specific nucleic acid sequence within the rpoB gene of M. tuberculosis.

Testing is carried out on the MTB/RIF test platform (GeneXpert, Cepheid), which integrates sample processing and PCR in a disposable plastic cartridge containing all reagents required for bacterial lysis, nucleic acid extraction, amplification, and amplicon detection. The only manual step is the addition of a bactericidal buffer to sample to be tested before transferring a defined volume to the cartridge. The MTB/RIF cartridge is then inserted into the GeneXpert device, which provides results within 2 hours [5].

Rifampicin resistance is taken as a surrogate marker for multidrug resistant (MDR) TB, the result of which is also rapidly provided by this test within 2 hours. This study was undertaken to evaluate the role of GeneXpert MTB/RIF in the diagnosis of clinically suspected EPTB since it was introduced as a diagnostic tool in our district since last two years.

\section{Materials and Methods}

A total of 241 samples from April 2016 to July 2018, from all clinically suspected EPTB patients with support of either laboratory or radiological evidence were studied.

Place of study: Basaveshwara Medical College and Hospital \& District General Hospital, Chitradurga district, Karnataka.

Type of study: Prospective analytical study

Sampling method: All continuous samples which satisfy inclusion criteria were taken for study.

Sample collection: Extra-pulmonary clinical samples were collected from hospitalized patients by treating physician after taking informed consent from all the patients. Based on the suspected site of localization, samples were collected under strict aseptic condition in appropriate quantity in sterile containers and sent to the central laboratory without any delay. These were pleural fluids ( $n=132)$, Lymph node FNAC $(n=59)$, CSF $(\mathrm{n}=21)$, pus $(\mathrm{n}=17)$, ascitic fluid $(\mathrm{n}=9)$ and synovial fluid $(n=3)$ specimens. All the samples were processed for biochemical, cytopathological examination and bacteriological examination using standard techniques as early as possible. If any delay is anticipated in processing, they were stored at $4^{\circ} \mathrm{C}$. Our study also included 20 controls i.e. cases not suspected of having EPTB. Demographic data and the necessary clinical details of the patients were collected from the patient's case sheets.

Inclusion criteria: (more than any two of the below to be fulfilled)

1. Clinical - fever and other constitutional symptoms for 2 weeks not responding to antibiotics

2. Biochemical - for CSF, proteins more than $60 \mathrm{mg} \%$, sugar $<2 / 3^{\text {rd }}$ of blood sugar

3. Pathological - for Body fluids, lymphocytosis $>60 \%$ or for Lymph node FNAC samples showing granulomatous picture.

4. Radiological - Chest X-ray/CT scan showing tubercular changes

5. Supportive evidence - family history of tuberculosis / positive tuberculin test

Exclusion criteria: All cases already on antitubercular treatment or had been confirmed as having Tuberculosis were excluded from the study.

Processing and interpretation of samples: The samples were screened for presence of acid fast bacilli by conventional $\mathrm{ZN}$ technique. The same samples were also used for testing by GeneXpert MTB/RIF Assay. According to standard operating procedure, sampling reagent (containing $\mathrm{NAOH}$ and isopropanol) was mixed with the sample in a ratio of $2: 1$, in a specimen container. The container was closed and shaken vigorously on a vortex mixer for 10 minutes. Then it was left at room temperature for 10 minutes after which it was once again shaken vigorously for 10 more minutes.

The sample in the container was left to stand at room temperature for 10 minutes. Finally $2 \mathrm{ml}$ of the processed specimen was taken in a sterile plastic disposable pipette and transferred to GeneXpert cartridge. The cartridge is loaded into the GeneXpert instrument, and an automatic process completes the remaining assay steps. The electronic results were sent directly from the GeneXpert test system to the central database. Lyophilized Bacillus globigii spores present in the assay cartridge serve as an internal process 


\section{Original Research Article}

control. Assays that found to be negative for M.tuberculosis and also negative for B.globigii internal control are reported as invalid tests. The test was repeated with the same mixture whenever an invalid result was found [6].

The results obtained were interpreted as follows [6]:

- MTB detected; RIF resistance detected (MTB+, RIF+)
- MTB detected; RIF resistance not detected $(\mathrm{MTB}+$, RIF-)

- MTB detected; RIF resistance indeterminate $(\mathrm{MTB}+$, RIF In)

- MTB not detected (MTB-)

Wherever possible, the patients were followed up to know their clinical status and the treatment outcome.

\section{Results}

Out of 241 EPTB patients, majorities (93.7\%) of patients were adults and only $6.22 \%$ were children. Maximum cases (107) were in the age group 30-49 years of age (Table 1). About139 (57.67\%) were male and $102(42.32 \%)$ were female (Table 2) with a male to female ratio of 1.36:1.Of 139 males, MTB was detected in12 (52.17\%) by GeneXpert and out of 102 females, MTB was detected in $11(47.82 \%)$ by GeneXpert. None of the patients were currently on antitubercular treatment (ATT).

241 samples of EPTB received in the Microbiology Laboratory for detecting MTB were categorized as listed in Table 3. Pleural fluid accounted for 54.7\% (132) of EPTB samples, followed by $24.4 \%$ (59) accounted for by Lymph node FNAC, followed by $8.7 \%$ (21) accounted for CSF and pus accounted for $7.0 \%$ (17). The remaining comprised of ascitic fluid $3.7 \%$ (9) and synovial fluids $1.2 \%$ (3). All samples were smear microscopy negative for AFB by ZN staining. MTB was detected in 23 (9.54\%) EPTB samplesby GeneXpert MTB/ RIF Assay. Among 132 pleural fluid samples, 8 (6.06\%) were positive for MTB by GeneXpert MTB/ RIF Assay, among 55 lymph node samples 8 (13.55\%) were positive, among 21 CSF samples $5(23.80 \%)$ were positive, among 17 pus samples $2(11.76 \%)$ were positive. Of the 23 MTB positive samples detected by GeneXpert MTB/ RIF Assay, all the samples were sensitive to rifampicin (Table 4).

Table-1: Showing Age distribution with positivity rate of GeneXpert MTB/RIF Assay

\begin{tabular}{|c|c|c|}
\hline AgeRange & No. of EPTB cases(\%) & No. of GeneXpert Positive (\%) \\
\hline $0-9$ yrs & $13(5.39 \%)$ & $3(23.07 \%)$ \\
\hline $10-29$ Yrs & $42(17.42 \%)$ & $6(14.28 \%)$ \\
\hline $30-49$ Yrs & $107(44.39 \%)$ & $12(11.21 \%)$ \\
\hline 50 yrs\&Above & $79(32.7 \%)$ & $2(2.53 \%)$ \\
\hline Total & $\mathbf{2 4 1}$ & $\mathbf{2 3 ( 9 . 5 4 \% )}$ \\
\hline
\end{tabular}

Table-2: Showing Co-relation of Sex with positivity rate of GeneXpert MTB/RIF Assay

\begin{tabular}{|c|c|c|}
\hline Sex & No. of EPTB Cases (\%) & No. of GeneXpert Positive (\%) \\
\hline Male & $139(57.67 \%)$ & $12(8.63 \%)$ \\
\hline Female & $102(42.32 \%)$ & $11(10.78 \%)$ \\
\hline Total & 241 & $23(9.54 \%)$ \\
\hline
\end{tabular}

Table-3: Showing GeneXpert MTB/RIF Assay in different types of EPTB specimens

\begin{tabular}{|c|c|c|c|c|}
\hline Specimen type & No (\%) & $\begin{array}{c}\text { No. of GeneXpert } \\
\text { Positives }\end{array}$ & $\begin{array}{c}\text { No. ofGeneXpert } \\
\text { Negatives }\end{array}$ & Sensitivity (\%) \\
\hline Pleural fluid & $132(54.77 \%)$ & 08 & 124 & $6.06 \%$ \\
\hline Lymph node FNAC & $59(24.81 \%)$ & 08 & 51 & $13.55 \%$ \\
\hline CSF & $21(8.71 \%)$ & 05 & 16 & $23.80 \%$ \\
\hline Pus & $17(7.05 \%)$ & 02 & 15 & $11.76 \%$ \\
\hline Ascitic fluid & $09(3.71 \%)$ & 00 & 09 & 00 \\
\hline Synovial fuid & $03(1.24 \%)$ & 00 & 03 & 00 \\
\hline Total & $\mathbf{2 4 1}$ & $\mathbf{2 3}$ & $\mathbf{2 1 8}$ & $\mathbf{9 . 5 4 \%}$ \\
\hline
\end{tabular}


Original Research Article

Table-4: Showing Rifampicin sensitivity results among MTB positive cases on GeneXpert MTB/RIF Assay

\begin{tabular}{|c|c|c|}
\hline No. of MTB positiveEPTB cases & Rifampicin SensitiveNo (\%) & Rifampicin Resistant No (\%) \\
\hline 23 & $23(100 \%)$ & $00(0 \%)$ \\
\hline
\end{tabular}

Table-5: Showing GeneXpert results among EPTB cases and controls.

\begin{tabular}{|c|c|c|}
\hline No. of cases & No. of GeneXpert Positive & No. of GeneXpert Negative \\
\hline Controls -20 & 00 & 20 \\
\hline EPTB cases -241 & 23 & 218 \\
\hline Total -261 & 23 & 238 \\
\hline
\end{tabular}

\section{Discussion}

Smear microscopy used to be most commonly used method for diagnosis of tuberculosis till very recently in most of the centers. With the continuation of using only microscopy as method for diagnosis of EPTB, there is every chance that many cases would be missed because EPTB cases are paucibacillary and these missed cases not put on anti-TB treatment would continue to spread the disease among the community. GeneXpert was installed at district hospital in our city with collaboration with RNTCP in March 2016. Since then all the clinically suspected tuberculosis cases are tested by this method.

In our study we found majority of EPTB cases $44.39 \%$ $(107 / 241)$ were of age group 30 to 49 years followed by $32.78 \%(79 / 241), 17.42 \%(42 / 241)$ were of 50 years $\&$ above age and 10-29 years respectively and least number of EPTB cases $5.39 \%$ (13/241) were found in 0-9 years of age group but maximum GeneXpert positivity rate i.e. $23.07 \%(3 / 13)$ for MTB detection found in 0-9 years of age followed by $14.28 \%$ (6/42), $11.21 \%(12 / 107)$ in $10-29$ years and $30-49$ years respectively and least GeneXpert positivity rate i.e. $2.53 \%(2 / 79)$ in 50years and above age group. The reasons that make this age group (30 to 49 years) vulnerable to TB are many. They are socially more active and are more exposed to open cases of TB than others. K. Arora et al [7] conducted a study on trends of EPTB and found higher detection of EPTB cases in younger age group.

In our study, out of 241 EPTB cases there were 139 $(57.67 \%)$ males and $102(42.32 \%)$ females. Male to Female ratio was 1.36:1. Likely reasons of male preponderance are as follows: a) In a male dominated society, usually he is the earning member. As he goes out for work, he is more likely to come in contact with an active TB case. b) Men are more likely to acquire habits like smoking and alcoholism which predispose to TB.Out of 139 males, MTB was detected in12 (52.17\%) and out of 102 females, MTB was detected in
11(47.82\%). These findings correlate with Gaur et al [8], in which they found $174(65.16 \%)$ males and 93(34.83\%) females. Of 174 males, MTB was detected in $26(14.9 \%)$ and out of 93 females MTB was detected in $21(22.5 \%)$.

In our study pleural fluid samples 132 (54.77\%) were most common followed by lymph node aspirate 59 (24.81\%), CSF21 (8.71\%), pus 17 (7.05\%), ascetic fluid $9(3.71 \%)$ and synovial fluid $3(1.24 \%)$ samples. This finding is similar to study conducted by Chanderet al [9], where pleural fluid samples found to be the most common i.e. 53 cases $(61.6 \%)$, followed by lymph node aspirates i.e. 20 cases $(23.2 \%)$ and ascitic fluid samples i.e. 8 cases $(9.3 \%)$. In another study done by Prakashet al [10] also the total number of different types of EPTB cases included pleural 148 (28.03\%), lymph node131 (24.81\%), abdomen 51(9.66\%), CNS66 (12.50\%), bones and joints 65 (12.31\%) and others 67 (12.69\%).

Overall $9.54 \%$ of EPTB samples were positive by GeneXpert MTB/RIF Assay in our study and is comparable to the results of Praveen et al [11], who reported $9.4 \%$ positivity. Causse et al [12] reported $11.8 \%$ positive GeneXpert results.

In a study conducted by Praveen et al [11], they found $10.1 \%$ plueral fluid sample positive for MTB by Gene Xpert and Hillemann et al[13] found 3 positive GeneXpert results out of 113 pleural fluid samples $(2.9 \%)$ all of which were negative on mycobacterial culture, the positivity rate for pleural fluids in our study was $6.06 \%(8 / 132)$ was in accordance with above studies. Rufai et al [14] showed that the Xpert has very low diagnostic sensitivity of $14.2 \%$ in pleural fluid, even in culture proven cases. Porcel et al [15] concluded that the Xpert MTB/RIF assay has a limited diagnostic capacity for pleural fluid samples of TB origin. This study found the sensitivity of Xpertin pleural fluid specimens to be very low, with more than half of all pleural TB patients being missed by this test. 


\section{Original Research Article}

Guidelines on EPTB for India recommend that Xpert MTB/RIF should not be used to diagnose pleural TB exclusively [16]. Our study found detection by Gene Xpert in CSF samples to be $23.80 \%$, which is higher than that in any other specimen type (lymph node, pleural fluid, pus, ascetic fluid and synovial fluid). In a study from India [17], the positivity rate with GeneXpert assay was $24.6 \%$ for CSF. Various Studies that assessed GeneXpert in CSF samples found a variable sensitivity of $20-86 \%$ [18, 19, 20, and 21].

Guidelines on EPTB for India recommend that GeneXpert may be used as an adjunctive test for TB meningitis (TBM). A negative GeneXpert result on a CSF specimen does not rule out TBM. The decision to give ATT should be based on clinical features and CSF profile [16]. Data from prior studies suggested a potential role for nucleic acid amplification tests in the diagnosis of TB from CSF and the results for GeneXpert here are in accordance with the findings. It's been observed that GeneXpert assay does not reach the sensitivity of culture, however it can significantly improve/ fasten the diagnosis of TBM especially in places where expertise for culture or other diagnostic tests are not available or where a rapid diagnosis of TB is necessary [11].

Our study found $13.55 \%$ GeneXpert detection rate in lymph node samples. Kumar et al [22] found higher positivity rate $66.7 \%$ by GeneXpert in lymph node samples. We found that GeneXpert can be useful in confirming a diagnosis in patients suspected of Lymph node TB when considered alongside the results of FNAC, but a negative Xpert test does not rule out lymph node TB. Guidelines on EPTB for India also recommend that Xpert MTB/RIF should be used as an additional test to conventional smear microscopy, culture, and cytology in FNAC specimens [16].

GeneXpert did not detect MTB in any of the nine ascetic fluid \& three synovial fluid samples in this study, suggesting that a negative Xpert result does not rule out Tuberculosis in above samples \& they must be investigated further using other phenotypic methods. This finding was in accordance with Kumar et al [22]. Previous studies have also reported low sensitivity $(6.3 \%, 9.3 \% 17.9 \%, 27.8 \%)$ of GeneXpert on ascitic fluid samples $[15,11,17,13] .7 .05 \%$ of pus samples were positive by Xpert MTB/RIF Assay in our study. The data on the utility of Xpert MTB/RIF for pus, synovial fluid are limited.

Out of 241 EPTB cases, none were found out be positive for $\mathrm{AFB}$ on the conventional $\mathrm{ZN}$ method in our study. There are various reports regarding the sensitivity of ZN smear for extra-pulmonary specimen ranging from as low as $0 \%$ to as high as $75 \%$. This limitation has been reported to be due to inadequacy and paucibacillary nature of extra pulmonary specimens [23]. Meenal Bagdia et al reported positivity rate of $9.28 \%$ on $\mathrm{ZN}$ method [24]. Out of 241 EPTB cases 23 (9.54\%) were found positive by GeneXpert in our study.So, GeneXpert was able to pick 23 EPTB cases which otherwise would have been missed by ZN smear examination alone.We also compared diagnostic modalities of pulmonary tuberculosis with EPTB. Out of a total of 1497 suspected Pulmonary TB cases (PTB), $199(13.3 \%)$ were found out be positive for MTB by sputum smear examination (ZN stain) whereas 266 (17.8\%) PTB cases were found positive by GeneXpert. So, GeneXpert was able to pick 67 PTB cases which otherwise would have been missed by sputum smear examination alone. The above result clearly shows that Gene Xpert is the only rapid diagnostic method currently available for detecting MTB in EPTB and PTB specimens.

Not only MTB detection but also rapidly determining the patient's multidrug-resistant tuberculosis (MDRTB) status in such cases is of prime importance in bringing to an end the spread of MDR-TB and decreasing mortality. There was no rifampicin resistance in the population studied, all the 23 MTB positive samples detected by Gene Xpert-MTB/RIF assay in our study were sensitive to Rifampicin. This finding was in accordance with Rufai et al [14], who have also reported similar sensitivity of rifampicin, whereas Pravin et al [11] detected Rifampicin resistance in 4 out of 16 MTB detected in the pleural fluid and one rifampicin resistance out of 5 MTB detected in the ascitic fluid samples.

Our study included 20 controls i.e. cases not suspected of having EPTB; MTB was not detected in any of the controls by GeneXpert assay. Hence no false positive results were detected in our study which indicates higher specificity of Gene Xpert in our study, The biggest advantage of GeneXpert is that of sample processing. The process is simplified into a very simple and single step. Data from a recent study confirm that the $\mathrm{MTB} / \mathrm{RIF}$ assay generates no infectious aerosols [25]. The simplicity and safety feature of this set up encourages us to use this as a rapid and highly sensitive method for detection of MTB cases as well as drug resistance in these cases without the need for sending the sample for higher centres and delaying the diagnosis. However the drawback of this set up is that it can process only four samples every two hours and also the cost can be too high for routine laboratories to afford. 


\section{Original Research Article}

Although GeneXpert assay is considered a breakthrough in the diagnosis of PTB and EPTB, one of the major limitations of this technique is that it cannot distinguish between viable and non-viable microorganisms while detecting MTB DNA. Hence it should not be used to monitor patients or efficacy of the treatment. Our study has following limitations (a) not having comparative data with Culture. (b) It was an experimental study and the samples included were also small to make a generalized statement.

\section{Conclusions}

In our study Gene Xpert was found to be more sensitive than routine smear methods. It detected many extrapulmonary samples to be positive for tuberculosis, all of which were missed by ZN staining. Also within a short duration it detected Rifampicin sensitivity. Due to the economical constraints in resource limited settings and those laboratories receiving large numbers of samples, judicious use of GeneXpert is recommended and can be used in Indian health-care setting only as an additional tool for the diagnosis of EPTB.

What this study adds to existing knowledge: The Gene Xpert MTB/RIF performance varies with the EPTB sample type. Although it has limited sensitivity, it was found to be simple, rapid and more effective method for detection of EPTB cases and also the drug resistance could be detected simultaneously, thus reducing the time taken for initiation of treatment. Xpert assay is relatively more expensive than traditional culture methods; however it makes an important contribution to the modern day detection of TB as it has higher sensitivity than smear and provides a more rapid diagnosis than culture and histology.

\section{Contribution from the Author}

1. Dr. Jagadevi: Data Collection, Analysis and preparation of Manuscript

2. Dr. Sumanta A:Data Collection, Analysis and Preparation of the Manuscript.

3. Dr. Shubha D S:Data collection, Analysis and Preparation of the Manuscript.

4. Dr. Sudhindra K S: Analysis and Preparation of the Manuscript.

5. Dr. Basavaraj Sangolli: Analysis and Preparation of the Manuscript.

Findings: Nil; Conflict of Interest: None initiated

Permission from IRB: Yes

\section{References}

1. Grange JM, Zumla AI. Tuberculosis. In: Manson's Tropical diseases. 22nd edition. Saunders, Elsevier; 2009. p.983-1038.

2. Avashia S, Bansal D, Ahuja K, Agrawal V. Comparison of conventional methods with gene xpertmtb/rif assay for rapid detection of mycobacterium tuberculosis and rifampicin resistance in extrapulmonary samples. Int J Med Res Rev 2016;4(2):181185. doi: 10.17511/ijmrr.2016.i02.010.

3. Hall GS. Primary processing of specimens and isolation and cultivation of mycobacteria. Clin Lab Med. 1996 Sep;16(3):551-67.

4. Park C, Hixon D, Furguson C. Rapid recovery of mycobacteria from clinical specimens using automated radiometric technique. Am J Clin Path 1984; 81: 341-5.

5. Piatek AS, Tyagi S, Pol AC, et al. Molecular beacon sequence analysis for detecting drug resistance in Mycobacterium tuberculosis. Nat Biotechnol. 1998 Apr;

16 (4): 359-63. DOI: 10. 1038/ nbt 0498359

6. World Health Organization (2011) Automated realtime nucleic acid amplification technology for rapid and simultaneous detection of tuberculosis and rifampicin resistance: Xpert MTB/RIF system. Policy statement.

7. Arora VK, Chopra KK. Extra pulmonary tuberculosis. Indian J Tuberc. 2007 Oct;54(4):165-7.

8. Gour SM, Munje RP, Babu NP, Sushant M, Jites A. Genotypic diagnosis of extra pulmonary tuberculosis CBNAAT a novel tool.MedPulse International Journal of Medicine. November 2017; 4(2): 79-82.doi: 10. 26611/1021425

9. Chander V, Raina SK, Bhardwaj AK, Kashyap S, Gupta AK, Sood A. Clinico-Epidemiological Profile of extra Pulmonary Tuberculosis: A Report from a High Prevalence State of Northern India. Public Health Research 2012, 2(6): 185-189.doi:10.5923/j. phr.20120 206.02

10. Prakasha SR, Suresh G, D'sa IP, et al. Mapping the pattern and trends of extrapulmonary tuberculosis. J Glob Infect Dis. 2013 Apr;5(2):54-9. doi: 10.4103/ 0974- 777X.112277. 


\section{Original Research Article}

11. Pravin KN and Chourasia E. Use of GeneXpert Assay for Diagnosis of Tuberculosis From Body Fluid Specimens, a 2 Years Study. J Microbiol Biotechnol, 2016, 1(1): 000105 .

12. Causse M, Ruiz P, Gutiérrez-Aroca JB, Casal M. Comparison of two molecular methods for rapid diagnosis of extrapulmonary tuberculosis. J Clin Microbiol. 2011 Aug;49(8):3065-7. doi: 10.1128/JCM. 00491-11. Epub 2011 Jun 8.

13. Hillemann D, Rüsch-Gerdes S, Boehme C, Richter E. Rapid molecular detection of extrapulmonary tuberculosis by the automated GeneXpert MTB/RIF system. DOI:10.1128/JCM.02268-10

14. Rufai SB, Singh A, Kumar P, et al. Performance of Xpert MTB/RIF Assay in Diagnosis of Pleural Tuberculosis by Use of Pleural Fluid Samples. J Clin Microbiol. 2015 Nov;53(11):3636-8. doi: 10.1128/JCM. 02182-15. Epub 2015 Aug 26.

15. Porcel JM, Palma R, Valdés L, et al. Xpert ${ }^{\circledR}$ $\mathrm{MTB} / \mathrm{RIF}$ in pleural fluid for the diagnosis of tuberculosis. Int $\mathrm{J}$ Tuberc Lung Dis. 2013 Sep;17 (9):1217-9. doi: 10.5588/ijtld.13.0178. Epub 2013 Jul 3.

16. Sharma SK, Ryan H, Khaparde S, et al. Index-TB guidelines: Guidelines on extrapulmonary tuberculosis for India. Indian J Med Res. 2017 Apr;145(4):448-463. doi: 10.4103/ijmr.IJMR_1950_16.

17. Alvarez-Uria G, Azcona JM, Midde M, et al. Rapid Diagnosis of Pulmonary and Extrapulmonary Tuberculosis in HIV-Infected Patients. Comparison of LED Fluorescent Microscopy and the GeneXpert MTB/RIF Assay in a District Hospital in India. Tuberc Res Treat. 2012;2012:932862. doi: 10.1155/2012/ 932862. Epub 2012 Aug 26.

18.Denkinger CM, Schumacher SG, Boehme CC, et al. Xpert MTB/RIF assay for the diagnosis of extrapulmonary tuberculosis: a systematic review and metaanalysis. Eur Respir J. 2014 Aug;44(2):435-46. doi: 10.1183/09031936.00007814. Epub 2014 Apr 2.

19. Tortoli E, Russo C, Piersimoni C, et al. Clinical validation of Xpert MTB/RIF for the diagnosis of extrapulmonary tuberculosis. Eur Respir J. 2012 Aug;40(2):442-7. doi: 10.1183/09031936.00176311. Epub 2012 Jan 12.

20. Nhu NT, Heemskerk D, Thu do DA, et al. Evaluation of GeneXpert MTB/RIF for diagnosis of tuberculous meningitis. J Clin Microbiol. 2014 Jan; 52
(1): 226-33. doi: 10.1128/JCM.01834-13. Epub 2013 Nov 6.

21. Patel VB, Theron G, Lenders L, et al. Diagnostic accuracy of quantitative PCR (Xpert MTB/RIF) for tuberculous meningitis in a high burden setting: a prospective study. PLoS Med. 2013 Oct; 10(10): e1001536. doi: 10.1371/journal.pmed.1001536. Epub 2013 Oct 22.

22. Kumar A, Das S, Paul DK. A Study on the Role of Cartridge Based Nucleic Acid Amplification Test (CBNAAT) for Diagnosing Pediatric Tuberculosis in a Tertiary Care Hospital in Eastern India. Acad J Ped Neonatol. 2018; 6(3): 555745. doi: 10.19080/AJPN. 2018.06.555745.

23. Munir MK, Shabbir I, Iqbal R, Khan SU. Comparison of AFB Smear Microscopy and Culture from Specimens Received for the Diagnosis of Extra Pulmonary Tuberculosis. P J M H S 2009; 1(1): 59-61.

24. Bagdia M, Bijwe S, Hirani N, Joshi A, Chowdhary A, Agrawal M, Bagdia A. Lab Diagnosis of Extra Pulmonary Tuberculosis: Comparison of Histopathology, Cytology, Zeihl Neelsen stain and Light Emission Diode Microscopy with Culture and Nucleic Acid Amplification Tests.Int J Cur Res Rev. 2018 Apr; 10 (8).15-19.

25. Banada PP, Sivasubramani SK, Blakemore R, et al. Containment of bioaerosol infection risk by the Xpert MTB/RIF assay and its applicability to point-of-care settings. J Clin Microbiol. 2010 Oct;48(10):3551-7. doi: 10.1128/JCM.01053-10. Epub 2010 Aug 18.

26. Denkinger CM, Schumacher SG, Boehme CC, et al. Xpert MTB/RIF assay for the diagnosis of extrapulmonary tuberculosis: a systematic review and metaanalysis. Eur Respir J. 2014 Aug; 44 (2): 435-46. doi: 10. 1183 / 09031936. 00007814. Epub 2014 Apr 2.

27. Tortoli E, Russo C, Piersimoni C, et al. Clinical validation of Xpert MTB/RIF for the diagnosis of extrapulmonary tuberculosis. Eur Respir J. 2012 Aug; 40 (2):442-7. doi: 10.1183/09031936.00176311. Epub 2012 Jan 12.

28. Nhu NT, Heemskerk D, Thu do DA, et al. Evaluation of GeneXpert MTB/RIF for diagnosis of tuberculous meningitis. J Clin Microbiol. 2014 Jan; 52 (1):226-33. doi: 10.1128/JCM.01834-13. Epub 2013 Nov 6. 


\section{Original Research Article}

29. Patel VB, Theron G, Lenders L, et al. Diagnostic accuracy of quantitative PCR (Xpert MTB/RIF) for tuberculous meningitis in a high burden setting: a prospective study. PLoS Med. 2013 Oct; 10(10): e1001536. doi: 10.1371/journal.pmed.1001536. Epub 2013 Oct 22.

30. Kumar A, Das S, Paul DK. A Study on the Role of Cartridge Based Nucleic Acid Amplification Test (CBNAAT) for Diagnosing Pediatric Tuberculosis in a Tertiary Care Hospital in Eastern India. Acad J PedNeonatol. 2018; 6(3): 555745. doi: 10.19080/AJPN. 2018. 06.555745.

31. Munir MK, Shabbir I, Iqbal R, Khan SU. Comparison of AFB Smear Microscopy and Culture from Specimens Received for the Diagnosis of Extra Pulmonary Tuberculosis. P J M H S 2009; 1(1):59-61.

32. Bagdia M, Bijwe S, Hirani N, Joshi A, Chowdhary A, Agrawal M, Bagdia A. Lab Diagnosis of Extra Pulmonary Tuberculosis: Comparison of Histopathology, Cytology, Zeihl Neelsen stain and Light Emission Diode Microscopy with Culture and Nucleic Acid Amplification Tests. Int J Cur Res Rev. 2018 Apr; 10 (8).15-19.

33. Banada PP, Sivasubramani SK, Blakemore R, et al. Containment of bioaerosol infection risk by the Xpert MTB/RIF assay and its applicability to point-of-care settings. J Clin Microbiol. 2010 Oct;48(10):3551-7. doi: 10.1128/JCM.01053-10. Epub 2010 Aug 18.

\section{How to cite this article?}

Jagadevi, Sumanta A, Shubha D.S, Sudhindra K.S, Sangolli B. Evaluation of GeneXpert MTB/RIF assay in diagnosis of extra pulmonary tuberculosis and rifampicin resistance. Trop J Path Micro 2018;4(7):478-485.doi:10.17511/jopm. 2018.i07.01. 